\title{
A set of proofs of the Collatz-Syracuse conjecture
}

\author{
Fausto Galetto
}

\begin{abstract}
Independent Researcher (past professor of Quality Management) Politecnico of Turin, Italy
*Corresponding Author: fausto.galetto@gmail.com
\end{abstract}

\begin{abstract}
We analyse some probabilistic methods for the proof of the conjecture and we provide a comparison of the proofs with another one found in the literature, using the Reliability Integral Theory and the SPQR Principle.

After, we show a new proof (probabilistic) by merging the infinite number of states into three SuperStates: the merged process is still a Markov process easily solvable.

Later we provide a different probabilistic proof of the Conjecture via the Reliability Integral Theory and the SPQR Principle. We devise a "ideal machine" (Gedanken Experiment) which, as quickly as one wants, makes transitions between the SuperStates and finally ends into the "Collatz Cycle" where it stays forever.

Eventually we show a non-probabilistic proof using Flow Graphs. This last method, based on the flow-graph theory, provides the means to solve completely the problem.

Having applied the SPQR ( $«$ Semper Paratus ad Qualitatem et Rationem») Principle, the author thinks that his methods either probabilistic or non_probabilistic are able to show the proof of the Collatz-Syracuse Conjecture.
\end{abstract}

Keywords Quality Methods, Numerical Methods, Hailstone Conjecture, Markov Processes, Reliability Integral Theory, Flow-graph Theory, SPQR.

\section{Introduction}

On January 2019 the author met the so called "the $3 x+1$ problem" (also named the $3 \mathrm{x}+1$ mapping and often called the Collatz Problem) in the mathematical site "maddmaths.simai.eu/category/rubriche/fake_papers" [1]; there he found the comments about a paper, named fake-paper, of Paul S. Bruckman A proof of the Collatz conjecture, International Journal of Mathematical Education in Science and Technology 39 (2008), no. 3, 403-407. [2] If one search that paper he found that it was retracted from publication.

The Collatz Problem was posed by L. Collatz in 1937; it states that the system of the two difference equations

$$
y_{k+1} \quad\left\{\begin{array}{c}
=\frac{1}{2} y_{k} \text { IF } y_{k} \text { is } E V E N \\
=3 y_{k}+1 \text { IF } y_{k} \text { is } O D D
\end{array}\right\}
$$

given the initial condition $\mathrm{y}_{0}=$ any integer positive number, arrives after some ( $\mathrm{n}$ is a number not known in advance) "continued" iterations at the value $\mathrm{y}_{\mathrm{n}}=1$. It is an innocent seeming arithmetic procedure applied to integers, while actually it is a very difficult problem to be solved: the Collatz conjecture remains today unsolved, as it has been for over 73 years.

It is considered a very difficult problem to be solved, in spite of its very simple definition; they say that Erdös commented that "Mathematics is not yet ready for such problems" and "Hopeless. Absolutely hopeless".

The Collatz algorithm has been numerically tested and found to always reach 1 for all numbers up to $2^{60}$ [double checked for convergence to 1].

Generally "the $3 x+1$ problem" is described by the Collatz function

$$
C(x)=\left\{\begin{array}{c}
3 x+1 \text { IF } x \equiv 1(\bmod 2) \\
x / 2 \text { IF } x \equiv 0(\bmod 2)
\end{array}\right\}
$$

and concerns the behaviour of this iterated function $\mathrm{C}(\mathrm{x})$, starting with any integer positive number $\mathrm{y}_{0}$.

$\mathbf{3}+\mathbf{1}$ Conjecture: Starting from any positive integer $y_{0}$, iterations of the function $C(x)$ will eventually reach the number 1 . Thereafter iterations will cycle, taking successive values $1,4,2,1, \ldots$.

The set $\{1,4,2\}$ is named Collatz Cycle.

Several scholars prefer to use a different function $\mathrm{T}(\mathrm{x})$

$$
T(x)=\left\{\begin{array}{c}
(3 x+1) / 2 \text { IF } x \equiv 1(\bmod 2) \\
x / 2 I F \quad x \equiv 0(\bmod 2)
\end{array}\right\}
$$

The conjecture is that for every integer $\mathrm{y}_{0}$ there exists a $\mathrm{k}$ such that $\mathrm{T}^{\mathrm{k}}\left(\mathrm{y}_{0}\right)=1 ; \mathrm{T}^{\mathrm{k}}()=\mathrm{T}\left[\mathrm{T}^{\mathrm{k}-1}()\right]$, iterated mapping.

We prefer to use the (1) formulation.

The system (1) can be reduced to a non-linear difference equation, as the following one

$$
4 y_{k+1}-7 y_{k}-2+(-1)^{y_{k}}\left[5 y_{k}+2\right]=0
$$

The numbers $\mathrm{y}_{\mathrm{k}+1}$ of the sequence provided by the previous (Collatz) equations are sometimes named hailstone numbers.

This problem goes under many other names, including the Collatz-Syracuse problem,...

After January 2019 the author (FG) studied the papers [3, $4,5]$ and later wrote his first document [6].

The document [6] is a "probabilistic proof" of the Conjecture, as it is [3]. The author's proof is based on the use 
of the stochastic processes given in his books $[7,8]$ and on the SPQR Principle [9].

Since [3] did not provided any probabilistic proof of the probability that the "Collatz Cycle" could be attained, the document [10] made a comparison between [3] and [5], showing the superiority of the last.

Other documents dealt with the Problem through the flow-graphs theory [11] and moreover merging the infinite states of the stochastic process into only 3 SuperStates [12], that obviously it's very easy analyzable.

The document [13] "Collatz-Syracuse Conjecture, proof by Reliability Integral Theory" uses the stochastic processes given in the books [7, 8] and on the SPQR Principle [9], applied to the 3 SuperStates [12].

This paper aims at showing this various ways of approaching the Collatz Problem making its solution "very probable"; it cannot be considered yet a Mathematical proof, but in any case we have to remember Erdös, a great mathematician, who commented that "Mathematics is not yet ready for such problems" and "Hopeless. Absolutely hopeless".

We end by mentioning Schorer, P. who claims some proofs of the Collatz Problem, but he regrets of not being taken seriously $[14,15,16]$.

\section{The Stochastic Model}

The Collatz Problem can be modelled by a "system making transitions between its states".

If we name "state of the system" the integer positive number generated by (1), we see that the problem is transformed into the following:

given any initial state $\mathrm{y}_{0}$, the system makes a certain number $\mathrm{n}$ of transitions ( $\mathrm{n}$ is a number not known in advance) and finally it ends into the state $\mathrm{y}_{\mathrm{n}}=1$.

Any state of the system is a vertex in a flow-graph. The rules (1) give the next state of the system i.e. the next vertex in the graph: the edge traversed at time $\mathrm{k}+1[$ the $\mathrm{k}+1$ time that we apply the rule (1)] which we name $e_{k+1}$ is $\left(y_{k}, y_{k+1}\right)$.

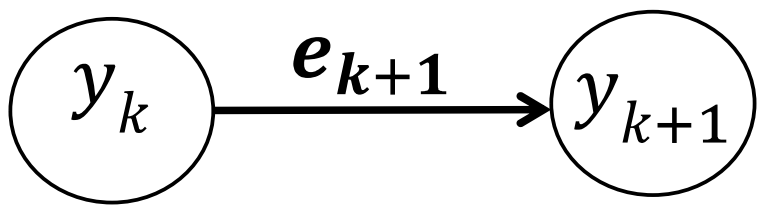

Figure 1. The system transition at time $k+1$

We can associate to any state of the system $\mathrm{y}_{\mathrm{k}}$ of the edge $\mathrm{e}_{\mathrm{k}+1}=\left(\mathrm{y}_{\mathrm{k}}, \mathrm{y}_{\mathrm{k}+1}\right)$ traversed at time $\mathrm{k}+1$ the index of the row of a matrix $\mathrm{P}$ and to state $\mathrm{y}_{\mathrm{k}+1}$ the index of the column of the same matrix $P$; then we can describe the graph of the system transitions by the matrix $\mathrm{P}$ with entries 1 related to the arrow of the transition $\mathrm{y}_{\mathrm{k}} \rightarrow \mathrm{y}_{\mathrm{k}+1}$ for any edge $\mathrm{e}_{\mathrm{k}+1}=\left(\mathrm{y}_{\mathrm{k}}\right.$, $\left.\mathrm{y}_{\mathrm{k}+1}\right)$.

Then for any state of the system $\mathrm{y}_{\mathrm{k}}$ there is an infinite dimensional row vector $\mathrm{u}(\mathrm{k})$, with all entries $\mathrm{u}_{\mathrm{i}}(\mathrm{k})=0$, but one entry $u_{\mathrm{y}}(\mathrm{k})=1$, related to the edge $\mathrm{e}_{\mathrm{k}+1}=\left(\mathrm{y}_{\mathrm{k}}, \mathrm{y}_{\mathrm{k}+1}\right)$ : it is a unit vector of a vector space. The vector $u(k)$ refers to the $\mathrm{k}$-th iteration of a mapping $\mathrm{C}$ : the result of the mapping $\mathrm{C}$ to the vector $\mathrm{u}(\mathrm{k})$ is denoted $\mathrm{u}(\mathrm{k}+1)=\mathrm{u}(\mathrm{k}) \mathrm{C}$. The vector $\mathrm{u}(\mathrm{k}+1)$ is unit vector with all entries $\mathrm{u}_{\mathrm{j}}(\mathrm{k}+1)=0$, but one entry $\mathrm{u}_{\mathrm{y} *}(\mathrm{k}+1)=1$, where we have the subindexes $\mathrm{y}^{* \neq \mathrm{y}}$. The subindexes are according to (1): if $\mathrm{u}_{\mathrm{y}}(\mathrm{k})=1$, then $\mathrm{y}=\mathrm{y}_{\mathrm{k}}$ and the index $\mathrm{y}^{*}$ of entry $\mathrm{u}_{\mathrm{y}^{*}}(\mathrm{k}+1)=1$ of the vector $\mathrm{u}(\mathrm{k}+1)$ has index $\mathrm{y}^{*}=\mathrm{y}_{\mathrm{k}} / 2$ IF $\mathrm{y}_{\mathrm{k}}$ is $\underline{\text { even }}$, and $\mathrm{y}^{*}=3 \mathrm{y}_{\mathrm{k}}+1$ IF $\mathrm{y}_{\mathrm{k}}$ is $\underline{\text { odd }}$.

The mapping $C$ [related to the graph] is provided by an infinite-dimensional matrix $\mathbf{P}=\left[\mathrm{a}_{\mathrm{ij}}\right]$, named transition matrix (with infinite rows and columns); rows and columns are indexed by the natural numbers (states of the system) 1 , $2,3,4, \ldots, n, n+1, \ldots$; every $\mathrm{a}_{\mathrm{ij}}$ entry is 0 , except

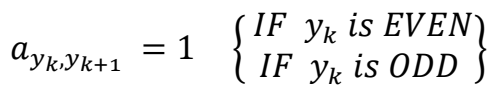

where the indexes $\mathrm{i}$ and $\mathrm{j}$ are given by (1), for the arrows $\mathrm{e}_{\mathrm{k}+1}=\left(\mathrm{y}_{\mathrm{k}}, \mathrm{y}_{\mathrm{k}+1}\right)$.

Accordingly we have

$$
\mathrm{u}(\mathrm{k}+1)=\mathrm{u}(\mathrm{k}) \mathrm{P}
$$

In the figure 2 we show the transition matrix $P$; the 3 by 3 matrix with rows and columns indexed by the numbers 1,2 , 4 , is highlighted due to its importance:

- when the system is in the state 1 , the next transition is to state $4: \mathbf{1} \Rightarrow \mathbf{4}$

- when the system is in the state 2 , the next transition is to state $1: \mathbf{2} \Rightarrow \mathbf{1}$

- when the system is in the state 4 , the next transition is to state $2: \mathbf{4} \Rightarrow \mathbf{2}$

All this means that when the system enters one of those 3 states [1, 2, 4] it never leaves them, the system (or the process) circulates in the set $\{1,2,4\}$ forever. It is a "periodic process".

The matrix $\mathrm{P}$ can be partitioned into 4 submatrices, written simply as

$$
P=\left[\begin{array}{cc}
P_{11} & 0 \\
P_{21} & P_{22}
\end{array}\right]
$$

where $\mathrm{P}_{11}$ and $\mathrm{P}_{22}$ are square matrices, given more explicitly by (notice that $\mathrm{P}_{11}$ refers to the states $1,2,4$ )

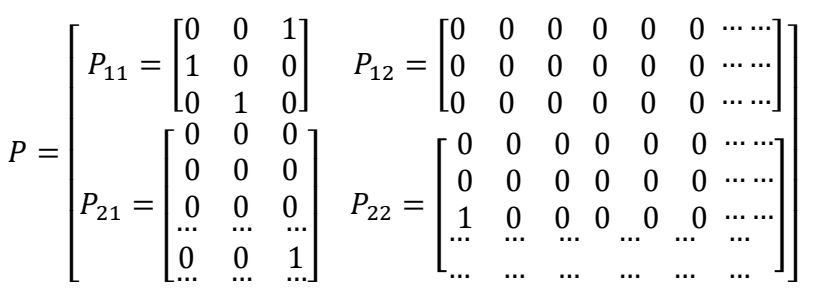

Notice that the submatrix $\mathrm{P}_{11}$ is orthogonal: its inverse is its transpose $P_{11}{ }^{-1}=P_{11}{ }^{T}$.

It is important to notice that $\mathrm{P}^{3}$, the $3^{\text {rd }}$ power of the matrix $\mathrm{P}$, is such that the submatrix

$$
P_{11}{ }^{3}=\left[\begin{array}{lll}
1 & 0 & 0 \\
0 & 1 & 0 \\
0 & 0 & 1
\end{array}\right]
$$


is the identity matrix; when the system reaches the set $\{1,2$, $4\}$ of the states it remains there forever. It follows that $P_{11}{ }^{-1}=P_{11}{ }^{2}=P_{11}{ }^{T}$.

The rectangular submatrix in the upper right corner has only 0 entries.

The $\mathrm{n}$-th power $\mathrm{P}^{\mathrm{n}}$ of the matrix $\mathrm{P}$ provides the transition probabilities, between the states $\mathrm{i} \Rightarrow \mathrm{j}$, in $\mathrm{n}$ steps: $p_{i, j}^{(n)}$ : is the conditional probability of entering the state $j$ at the nth step, given the initial state $\mathrm{i}$.

We have (see figures 2 and 3 )

$$
P^{n}=\left[\begin{array}{cc}
P_{11}^{n} & 0 \\
V_{n} & P_{22}{ }^{n}
\end{array}\right]
$$

The submatrix $V_{n}$ is related to the transitions to the set $S_{1}$ in $\mathrm{n}$ steps.

The $\mathbf{3 x + 1}$ Conjecture then can be stated as: There is a power $n$ such that, after $n$, the system will cycle within the Collatz Cycle $\{1,4,2\}$.

\begin{tabular}{|c|c|c|c|c|c|c|c|c|c|c|c|c|c|c|c|c|c|c|c|c|c|}
\hline state & 1 & 2 & 4 & 3 & 5 & 6 & 7 & 8 & 9 & 10 & 11 & 12 & 13 & 14 & 15 & 16 & 17 & 18 & 19 & 20 & $\ldots$ \\
\hline 1 & 0 & 0 & 1 & 0 & 0 & 0 & 0 & 0 & 0 & 0 & 0 & 0 & 0 & 0 & 0 & 0 & 0 & 0 & 0 & 0 & \\
\hline 2 & 1 & 0 & 0 & 0 & 0 & 0 & 0 & 0 & 0 & 0 & 0 & 0 & 0 & 0 & 0 & 0 & 0 & 0 & 0 & 0 & \\
\hline 4 & 0 & 1 & 0 & 0 & 0 & 0 & 0 & 0 & 0 & 0 & 0 & 0 & 0 & 0 & 0 & 0 & 0 & 0 & 0 & 0 & \\
\hline 3 & 0 & 0 & 0 & 0 & 0 & 0 & 0 & 0 & 0 & 1 & 0 & 0 & 0 & 0 & 0 & 0 & 0 & 0 & 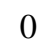 & 0 & \\
\hline 5 & 0 & 0 & 0 & 0 & 0 & 0 & 0 & 0 & 0 & 0 & 0 & 0 & 0 & 0 & 0 & 1 & 0 & 0 & 0 & 0 & \\
\hline 6 & 0 & 0 & 0 & 1 & 0 & 0 & 0 & 0 & 0 & 0 & 0 & 0 & 0 & 0 & 0 & 0 & 0 & 0 & 0 & 0 & \\
\hline 7 & 0 & 0 & 0 & 0 & 0 & 0 & 0 & 0 & 0 & 0 & 0 & 0 & 0 & 0 & 0 & 0 & 0 & 0 & 0 & 0 & \\
\hline 8 & 0 & 0 & 1 & 0 & 0 & 0 & 0 & 0 & 0 & 0 & 0 & 0 & 0 & 0 & 0 & 0 & 0 & 0 & 0 & 0 & \\
\hline 9 & 0 & 0 & 0 & 0 & 0 & 0 & 0 & 0 & 0 & 0 & 0 & 0 & 0 & 0 & 0 & 0 & 0 & 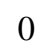 & 0 & 0 & \\
\hline 10 & 0 & 0 & 0 & 0 & 1 & 0 & 0 & 0 & 0 & 0 & 0 & 0 & 0 & 0 & 0 & 0 & 0 & 0 & 0 & 0 & \\
\hline 11 & 0 & 0 & 0 & 0 & 0 & 0 & 0 & 0 & 0 & 0 & 0 & 0 & 0 & 0 & 0 & 0 & 0 & 0 & 0 & 0 & \\
\hline 12 & 0 & 0 & 0 & 0 & 0 & 1 & 0 & 0 & 0 & 0 & 0 & 0 & 0 & 0 & 0 & 0 & 0 & 0 & 0 & 0 & \\
\hline 13 & 0 & 0 & 0 & 0 & 0 & 0 & 0 & 0 & 0 & 0 & 0 & 0 & 0 & 0 & 0 & 0 & 0 & 0 & 0 & 0 & \\
\hline 14 & 0 & 0 & 0 & 0 & 0 & 0 & 1 & 0 & 0 & 0 & 0 & 0 & 0 & 0 & 0 & 0 & 0 & U & U & 0 & \\
\hline 15 & 0 & 0 & 0 & 0 & 0 & 0 & 0 & 0 & 0 & 0 & 0 & 0 & 0 & 0 & 0 & 0 & 0 & 0 & 0 & 0 & \\
\hline 16 & 0 & 0 & 0 & 0 & 0 & 0 & 0 & 1 & 0 & 0 & 0 & 0 & 0 & 0 & 0 & 0 & 0 & 0 & 0 & 0 & \\
\hline 17 & 0 & 0 & 0 & 0 & 0 & 0 & 0 & 0 & 0 & 0 & 0 & 0 & 0 & 0 & 0 & 0 & 0 & 0 & 0 & 0 & \\
\hline 18 & 0 & 0 & 0 & 0 & 0 & 0 & 0 & 0 & 1 & 0 & 0 & 0 & 0 & 0 & 0 & 0 & 0 & 0 & 0 & 0 & \\
\hline 19 & 0 & 0 & 0 & 0 & 0 & 0 & 0 & 0 & 0 & 0 & 0 & 0 & 0 & 0 & 0 & 0 & 0 & U & U & 0 & \\
\hline 20 & 0 & 0 & 0 & 0 & 0 & 0 & 0 & 0 & 0 & 1 & 0 & 0 & 0 & 0 & 0 & 0 & 0 & 0 & 0 & 0 & \\
\hline$\ldots$ & 0 & 0 & 0 & 0 & 0 & 0 & 0 & 0 & 0 & 0 & 0 & 0 & 0 & 0 & 0 & 0 & 0 & 0 & 0 & 0 & \\
\hline$\ldots$ & 0 & 0 & 0 & 0 & 0 & 0 & 0 & 0 & 0 & 0 & 1 & 0 & 0 & 0 & 0 & 0 & 0 & 0 & 0 & 0 & \\
\hline$\ldots$ & 0 & 0 & 0 & 0 & 0 & 0 & 0 & 0 & 0 & 0 & 0 & 0 & 0 & 0 & 0 & 0 & 0 & 0 & 0 & 0 & \\
\hline$\ldots$ & 0 & 0 & 0 & 0 & 0 & 0 & 0 & 0 & 0 & 0 & 0 & 1 & 0 & 0 & 0 & 0 & 0 & U & U & 0 & \\
\hline$\ldots$ & 0 & 0 & 0 & 0 & 0 & 0 & 0 & 0 & 0 & 0 & 0 & 0 & 0 & 0 & 0 & 0 & 0 & 0 & 0 & 0 & \\
\hline$\ldots$ & 0 & 0 & 0 & 0 & 0 & 0 & 0 & 0 & 0 & 0 & 0 & 0 & 1 & 0 & 0 & 0 & 0 & U & U & 0 & \\
\hline$\ldots$ & 0 & 0 & 0 & 0 & 0 & 0 & 0 & 0 & 0 & 0 & 0 & 0 & 0 & 0 & 0 & 0 & 0 & 0 & 0 & 0 & \\
\hline$\ldots$ & 0 & 0 & 0 & 0 & 0 & 0 & 0 & 0 & 0 & 0 & 0 & 0 & 0 & 1 & 0 & 0 & 0 & 0 & 0 & 0 & \\
\hline$\ldots$ & 0 & 0 & 0 & 0 & 0 & 0 & 0 & 0 & 0 & 0 & 0 & 0 & 0 & 0 & 0 & 0 & 0 & 0 & 0 & 0 & \\
\hline$\cdots$ & 0 & 0 & 0 & 0 & 0 & 0 & 0 & 0 & 0 & 0 & 0 & 0 & 0 & 0 & 1 & 0 & 0 & 0 & 0 & 0 & \\
\hline$\ldots$ & 0 & 0 & 0 & 0 & 0 & 0 & 0 & 0 & 0 & 0 & 0 & 0 & 0 & 0 & 0 & 0 & 0 & U & U & 0 & \\
\hline$\ldots$ & 0 & 0 & 0 & 0 & 0 & 0 & 0 & 0 & 0 & 0 & 0 & 0 & 0 & 0 & U & 1 & 0 & U & U & 0 & \\
\hline
\end{tabular}

Figure 2. . The transition matrix P [only a part is shown], related to the flow-graph

In every row there is only one number 1 , while all the others are 0 . Therefore any row is a probability vector. 
This idea is important because we can consider the matrix $\mathrm{P}$ as a transition probability matrix, with the same properties of the matrices used for modelling the Markov Chains.

To take advantage of the Reliability Integral Theory (RIT) we arrange the rows and columns in the following order: 1, $2,4,3,5,6, \ldots . ., n, n+1, \ldots \ldots \ldots . .$. rows and columns 3 and 4 are exchanged as the reader can see in the figure 2 , where it is presented a part of the infinite matrix, with its entries.

The $\mathbf{a}_{\mathrm{ij}}=1$ entries mean that the Markov Process makes a transition from the state $\mathrm{i}$ to the state $\mathrm{j}$, with probability 1 . The matrix is named $\mathrm{P}$ and its entries are indicated $\mathbf{p}_{\mathbf{i j}}$ (to remember "probability").

Our system has a total number of states $\mathrm{N}=\infty$ : we indicate a $S$ the set of all the states. $S$ can be partitioned into two disjoints sets $\underline{S}_{\underline{1}}$, the set $\{1,2,4\}$ [of the periodic process] of the $\mathrm{N}_{1}=3$ states, and $\underline{S}_{2}$, the set $\{3,5,6, \ldots, \mathrm{n}, \mathrm{n}+1, \ldots$.$\} of the$ $\mathrm{N}_{2}=\infty$ states, $\mathrm{S}=\mathrm{S}_{1} \cup \mathrm{S}_{2}$ with $\mathrm{S}_{1} \cap \mathrm{S}_{2}=\varnothing: \mathrm{N}=\mathrm{N}_{1}+\mathrm{N}_{2}$.

See the figure 3 .

We partition the matrix $\mathrm{P}$ into four submatrices $\mathrm{P}_{11}, \mathrm{P}_{12}$, $\mathrm{P}_{21}, \mathrm{P}_{22}$, related to the transitions $\mathrm{i} \rightarrow \mathrm{j}\left(\mathrm{i} \in \mathrm{S}_{1}, \mathrm{j} \in \mathrm{S}_{1}\right)$ within the set $S_{1}=\{1,2,4\}$, related to the transitions $i \rightarrow j\left(i \in S_{1}, j \in\right.$ $S_{2}$ ) from $S_{1}=\{1,2,4\}$ to $S_{2}=\{3,5,6, \ldots, n, n+1, \ldots$.$\} , related$ to the transitions $i \rightarrow j\left(i \in S_{2}, j \in S_{1}\right)$ from $S_{2}=\{3,5,6, \ldots, n$, $n+1, \ldots$.$\} to S_{1}=\{1,2,4\}$ and related to the transitions $i \rightarrow j$ $\left(i \in S_{2}, j \in S_{2}\right)$ within the set $S_{2}=\{3,5,6, \ldots, n, n+1, \ldots$.$\} .$

You can see part of the submatrices in the formula (3); only the left upper submatrix is written in full, as one can see the set $S_{1}$.

The entries of such submatrices are related to the figure 2 .

The periodic process" circulating in the set $S_{1}=\{1,2,4\}$ is ruled by the submatrix $\mathrm{P}_{11}$.

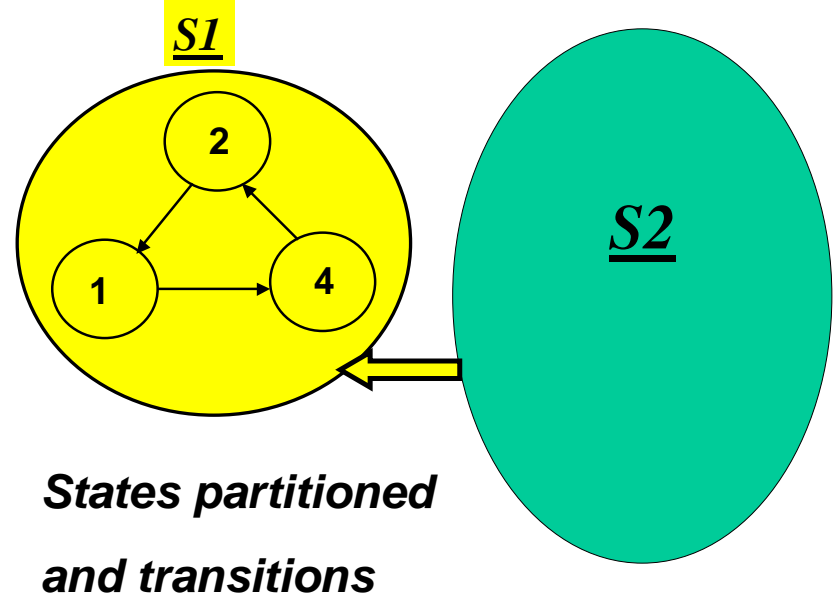

Figure 3. The two disjoint sets

\section{The Probabilistic Proof}

Now we consider the theory given in the books Reliability and Maintenance, Scientific Methods, Practical Approach, Volume one and two. $[7,8]$

There, two vectors $z \alpha_{1}$ and $z \alpha_{2}$ are defined, as follows,

- $\mathrm{z} \alpha_{1}$ is the vector of the (steady state) probabilities of entering into one of the states $\{1,2,4\}\left[\in \mathrm{S}_{1}\right]$, when there is a transition $\mathrm{S}_{2}=\{3,5,6, \ldots, \mathrm{n}, \mathrm{n}+1, \ldots.\} \Rightarrow$ $\mathrm{S}_{1}=\{1,2,4\}$.

- $\mathrm{z \alpha}_{2}$ is the vector of the (steady state) probabilities of entering into one of the states $\{3,5,6, \ldots, n, n+1, \ldots$. $\left[\in \mathrm{S}_{2}\right]$, when there is a transition from $\mathrm{S}_{1}=\{1,2,4\} \Rightarrow$ $\mathrm{S}_{2}=\{3,5,6, \ldots, \mathrm{n}, \mathrm{n}+1, \ldots$.$\} .$

$\mathrm{z} \alpha_{1}$ is by definition a three-dimensional row vector $[0,0$, 1] related to the set $S_{1}$; see the figure 3 . The system enters into the set $S_{1}$ only through the state 4 .

After entering $S_{1}$ the probability of being in the states $\{1$, $2,4\}$ is given by a vector $\pi=[1 / 3,1 / 3,1 / 3]$ solution of the relationship $\pi=\pi \mathbf{P}_{\mathbf{1 1}}$.

$\mathrm{z \alpha}_{2}$ is by definition an infinite dimensional row $\underline{\text { null }}$ vector $[0,0,0, \ldots ., 0, \ldots]$ related to the set $S_{2}$; see the figure 3. The system never enters into the set $S_{2}$ from $S_{1}$.

This means that (see figure 3 )

$>$ IF a person chooses a number $y_{0} \in S_{2}=\{3,5$, $6, \ldots, n, n+1, \ldots$.

$>$ and applies the rules $y_{k+1}\left\{\begin{array}{c}=\frac{1}{2} y_{k} \text { IF } y_{k} \text { is EVEN } \\ =3 y_{k}+1 \text { IF } y_{k} \text { is ODD }\end{array}\right\}$

$>$ then the Markov system enters the state $\mathrm{y}_{0} \in$ $\mathrm{S}_{2}$ for the first time,

$>$ it makes all the needed transitions within the set $\mathrm{S}_{2}$

$>$ until it goes into the set $S_{1}=\{1,2,4\}$ the Collatz Cycle

$>$ and it remains there, with probability vector $\pi$ : periodic process

So we see that there is probability 1 of entering the Collatz Cycle.

If we want, by making the state 1 an absorbing state the system stays in the state 1 , forever.

The hailstone numbers $\mathrm{y}_{\mathrm{k}+1}$ of the sequence provided by the Collatz equations (1) end into the number 1 , whichever is the starting number $\mathrm{y}_{0}$.

\section{Another Probabilistic Proof [3]}

Now we present shortly the ideas of $\mathrm{T}$. Carletti, D. Fanelli, 2016, Quantifying the degree of average contraction of Collatz orbits. arXiv:1612.07820.v1 [3]

The two authors claim to

... provide a novel argument to support the validity of the Collatz conjecture. To anticipate our findings, we shall demonstrate that the third iterate $T^{3}$ of the Collatz map admits three fixed points, 1, 2 and 4. These latter elements define the, supposedly unique, attracting cycle conjectured by Collatz. The third iterate map is naturally defined on the mod8 congruence classes of positive integers. We thus quantify the factor of relative compression or expansion, as follows the application of $T^{3}$, on each of the eight congruence classes obtained under the mod8 operation. we show that orbits are on average bound to asymptotically 
shrink in size so heading towards the deputed equilibrium. We will further enhance the resolution ... working on the congruence classes mod $8^{m}$ for any chosen $m$. Working in this generalised setting, we will prove that the average Collatz dynamics is contracting, for $m$ large as sought, namely shrinking the congruence classes arbitrarily close to the singletons corresponding to each integer.

To guide the reader through the text we shall hereafter provide a schematic outline of the main steps involved in the analysis, by making explicit reference to specific key results.

We give the information connecting the "boxed statements" with the Introduction and section 2:

The mentioned mapping $\mathrm{T}$ is represented (in the Introduction) by the Matrix P.

The mentioned "third iterate of the Collatz map hereby named $S=T^{03 \prime \prime}$ is represented (in the Introduction) by the Matrix $\mathrm{P}^{3}$.

The infinite matrix $\mathrm{P}^{3}$ is reduced to a stochastic matrix Q* (a matrix 8 by 8 ), using a congruence $\bmod 8$ on the integer numbers. Then 8 equivalence classes are obtained $\mathrm{B}(\mathrm{i}, 8)$ and indicated as $\mathrm{B}(0,8), \mathrm{B}(1,8), \mathrm{B}(2,8), \mathrm{B}(3,8)$, $\mathrm{B}(4,8), \mathrm{B}(5,8), \mathrm{B}(6,8), \mathrm{B}(7,8)$.

$$
Q^{*}=\left(\begin{array}{cccccccc}
\frac{1}{8} & \frac{1}{8} & \frac{1}{8} & \frac{1}{8} & \frac{1}{8} & \frac{1}{8} & \frac{1}{8} & \frac{1}{8} \\
0 & \frac{1}{4} & 0 & \frac{1}{4} & 0 & \frac{1}{4} & 0 & \frac{1}{4} \\
\frac{1}{4} & 0 & \frac{1}{4} & 0 & \frac{1}{4} & 0 & \frac{1}{4} & 0 \\
\frac{1}{2} & 0 & 0 & 0 & \frac{1}{2} & 0 & 0 & 0 \\
\frac{1}{4} & 0 & \frac{1}{4} & 0 & \frac{1}{4} & 0 & \frac{1}{4} & 0 \\
\frac{1}{4} & 0 & \frac{1}{4} & 0 & \frac{1}{4} & 0 & \frac{1}{4} & 0 \\
0 & \frac{1}{4} & 0 & \frac{1}{4} & 0 & \frac{1}{4} & 0 & \frac{1}{4} \\
0 & 0 & \frac{1}{2} & 0 & 0 & 0 & \frac{1}{2} & 0
\end{array}\right)
$$

Figure 4. The transition matrix, 8 by 8 , from the paper "Quantifying ... of Collatz orbits. arXiv:1612.07820.v1"

The matrix $\mathrm{Q}^{*}$ is given in figure 4 [rows and columns are indexed by the classes $\mathrm{B}(\mathrm{i}, 8)$ ]

The two authors show that there is a steady state probability vector, $\mathrm{P}_{\text {stat }}$, of being in the equivalence classes $B(i, 8): P_{\text {stat }}=[1 / 6,1 / 12,1 / 6,1 / 12,1 / 6,1 / 12,1 / 6,1 / 12]$

From $\mathrm{P}_{\text {stat }}$ it is clear that the process does arrive into the set $\{\mathrm{B}(1,8), \mathrm{B}(2,8), \mathrm{B}(4,8)\}$ BUT it does not stay there "forever" after its arrival. The process does not end in the set $\{1,2,4\}$, that is, process does not end in the "three fixed points, 1, 2 and 4." (as the authors say)! Therefore the authors are forced to prove that "that the deterministic trajectories are on average contracting."

Eventually they write in their Conclusion:

In this paper we have provided an analytical argument to support the validity of the so called Collatz conjecture, a long standing problem in mathematics which dates back to 1937. The analysis builds on three main pillars. In short, we (i) introduced the (forward) third iterate of the Collatz map (so to reduce the analysis of the period 3 cycle to a search for a fixed point) and considered the equivalence classes of integer numbers modulo 8; (ii) defined a Markov chain (based on a suitable non trivial measure) which runs on a set of finite states and whose transition probabilities reflect the deterministic map; (iii) showed that orbits are on average contracting, as follows strict bound that combines the visiting frequencies, as derived in the framework of the aforementioned stochastic picture, and the contraction/expansion factors associated to each transition among classes.

We rearrange the states [8 equivalence classes are obtained $\mathrm{B}(\mathrm{i}, 8)$ ] of the finite process described by the previous stochastic matrix $\mathrm{Q}^{*}$ (given in figure 4) as follows

\begin{tabular}{|c|c|c|c|c|c|c|c|c|}
\hline$\frac{\text { Matrix }}{\underline{\mathbf{Q}_{\mathbf{G}}}}$ & $\mathrm{B}(\mathbf{1 , 8})$ & $\mathrm{B}(2,8)$ & $\mathrm{B}(4,8)$ & $\mathrm{B}(\mathbf{5 , 8})$ & $\mathrm{B}(6,8)$ & $\mathrm{B}(\mathbf{0 , 8})$ & $\mathrm{B}(3,8)$ & B(7 8) \\
\hline $\mathrm{B}(\mathbf{1 , 8})$ & 0.25 & 0.00 & 0.00 & 0.25 & 0.00 & 0.00 & 0.25 & 0.25 \\
\hline $\mathrm{B}(2.8)$ & 0.00 & 0.25 & 0.25 & 0.00 & 0.25 & 0.25 & 0.00 & 0.00 \\
\hline$B(4.8)$ & 0.00 & 0.25 & 0.25 & 0.00 & 0.25 & 0.25 & 0.00 & 0.00 \\
\hline $\mathrm{B}(5.8)$ & 0.00 & 0.25 & 0.25 & 0.00 & 0.25 & 0.25 & 0.00 & 0.00 \\
\hline $\mathrm{B}(6.8)$ & 0.25 & 0.00 & 0.00 & 0.25 & 0.00 & 0.00 & 0.25 & 0.25 \\
\hline $\mathbf{B}(\mathbf{0 . 8})$ & 0.125 & 0.125 & 0.125 & 0.125 & 0.125 & 0.125 & 0.125 & 0.125 \\
\hline $\mathrm{B}(\mathbf{3 . 8})$ & 0.00 & 0.00 & 0.50 & 0.00 & 0.00 & 0.50 & 0.00 & 0.00 \\
\hline $\mathrm{B}(7.8)$ & 0.00 & 0.50 & 0.00 & 0.00 & 0.50 & 0.00 & 0.00 & 0.00 \\
\hline
\end{tabular}

where the states $\mathrm{B}(1,8), \mathrm{B}(2,8), \mathrm{B}(4,8)$ are in the left top corner.

The new Pstat is consequently

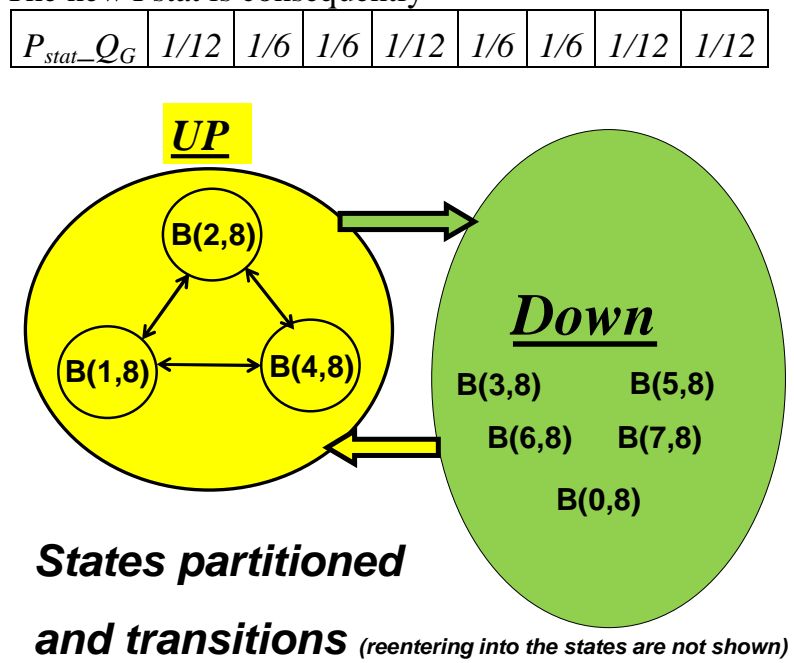

Figure 5. The two disjoint sets UP and Down (re-entering into the states are not shown)

The MTTF (Mean Time To Forward, from each UP state 
to the Down set) is a 3-dimensional column vector

$$
\mathrm{MTTF}=[1.333,2.0,2.0]^{\mathrm{T}}
$$

while the MTTR (Mean Time To Return, from each Down state to the UP set) is a 5-dimensional column vector MTTR $=[2.316,2.737,2.526,2.263,2.368]^{\mathrm{T}}$

MTTF and MTTR provide the mean number of transitions from each state of a set tot the disjoint set.

By using the theory given in the books [7, 8] related to Reliability Integral Theory [RIT] one can find two vectors $\mathrm{z} \alpha_{1}$ and $\mathrm{z} \alpha_{2}$ defined, as follows,

- $z \alpha_{1}$ is the vector of the (steady state) probabilities of entering into one of the UP, when there is a transition Down $\Rightarrow$ UP.

- $\mathrm{z \alpha}_{2}$ is the vector of the (steady state) probabilities of entering into one of the Down states, when there is a transition from UP $\Rightarrow$ Down.

$\mathrm{z \alpha}_{1}$ is a three-dimensional row vector related to the UP set (see the figure 5):

\begin{tabular}{|l|l|l|l|}
\hline$z \alpha_{1}$ & 0.27273 & 0.36364 & 0.36364 \\
\hline
\end{tabular}

so that the MUT (Mean Up Time) is MUT $=1.818182$

$\mathrm{zO}_{2}$ is a five-dimensional row vector related to the Down set (see the figure 5):

\begin{tabular}{|l|l|l|l|l|l|}
\hline$z \alpha_{2}$ & 0.09091 & 0.36364 & 0.36364 & 0.09091 & 0.09091 \\
\hline
\end{tabular}

so that the MDT (Mean Down Time) is MDT $=2.545455$

The MCT (Mean Cycle Time) is MCT $=4.363636$

The probability that the process is in the set $\mathrm{UP}=\{\mathrm{B}(1,8)$, $\mathrm{B}(2,8), \mathrm{B}(4,8)\}$ is $\mathrm{A}_{\mathrm{SS}}=0.42=\mathrm{MUT} / \mathrm{MCT}$ (as it must be).

Each state has its own recurrence time [with the fraction of time spent in each state (one of the 8 equivalent classes)]:

\begin{tabular}{|c|c|c|c|c|c|c|c|c|}
\hline state & $B(1)$ & $B(2)$ & $B(4)$ & $B(5)$ & $B(6)$ & $B(0)$ & $B(3)$ & $B(7)$ \\
\hline $\begin{array}{c}\text { recurrence } \\
\text { time }\end{array}$ & 12 & 6 & 6 & 12 & 6 & 6 & 12 & 12 \\
\hline $\begin{array}{c}\text { Time in } \\
\text { state }\end{array}$ & $\begin{array}{c}0.08 \\
33\end{array}$ & $\begin{array}{c}0.16 \\
67\end{array}$ & $\begin{array}{c}0.16 \\
67\end{array}$ & $\begin{array}{c}0.08 \\
33\end{array}$ & $\begin{array}{c}0.16 \\
67\end{array}$ & $\begin{array}{c}0.16 \\
67\end{array}$ & $\begin{array}{c}0.08 \\
33\end{array}$ & $\begin{array}{c}0.08 \\
33\end{array}$ \\
\hline
\end{tabular}

So we see that to "prove" the Collatz Conjecture one should prove that $\mathrm{A}_{\mathrm{SS}\{1,2,4\}}=1$ and $\mathrm{MUT}_{\{1,2,4\}}=\infty$, from the above ideas ...

The two authors (T. Carletti, D. Fanelli [3]) then consider a number $8^{\mathrm{m}}$ of equivalent classes $\mathrm{B}\left(\mathrm{i}, 8^{\mathrm{m}}\right)$, generated by the congruence $\bmod 8^{\mathrm{m}}$. By increasing $\mathrm{m}$, one generates more and more classes [process states], always with the Collatz cycle $\{1 ; 2 ; 4\}$.

The two authors prove that there is a stochastic matrix $\mathrm{Q}(\mathrm{m})$ [a $8^{\mathrm{m}}$ by $8^{\mathrm{m}}$ matrix] which provides a stationary row vector $P_{\text {stat }}(m)=[a, b, a, b, \ldots, a, b]$ solution of the equation $\mathrm{P}_{\text {stat }}(\mathrm{m})=\mathrm{P}_{\text {stat }}(\mathrm{m}) \mathrm{Q}(\mathrm{m}), \quad$ where $\quad \mathrm{a}=0.1667 / 8^{\mathrm{m}-1}$ and $\mathrm{b}=0.0833 / 8^{\mathrm{m}-1}$.

Again we see that to "prove" the Collatz Conjecture one should prove that $\mathrm{A}_{\mathrm{SS}\{1,2,4\}}=1$ and $\operatorname{MUT}_{\{1,2,4\}}=\infty$, from the above ideas ...

In F. Galetto opinion, the Collatz Conjecture is not made plausible by T. Carletti and D. Fanelli [1], because as $m \rightarrow \infty$ the probability that the process is in the Collatz cycle $\{1 ; 2$; 4 \} tends to 0 and not to $1 \ldots$ Perhaps F. Galetto did not understand...
Then the following remarkable conclusion ... is in doubt ...

The remarkable conclusion is therefore that the third iterate of the Collatz map is always contracting, when seen on the equivalence classes $B\left(i ; 8^{m}\right)$, for $m$ large as sought, and that the estimated bound for the contraction factor is independent on the classes index m. In other words, we can make the number of classes as large as wished (and consequently reduce their size so to approach the singletons with arbitrary accuracy), while still detecting a contracting deterministic dynamics, with a constant (independent on $\mathrm{m}$.) bound for the rate of contraction.

\section{The 3 SuperStates Model}

We go on with the probabilistic model related to the sections $1,2,3$.

We re-arrange the (infinite) transition probability matrix $\mathrm{P}$ (see figure 2) with a SuperState $\mathrm{SS}_{0}$ made of the 3 states $[1,2,4]$, a SuperState $\mathrm{SS}_{1}$ made of the infinite EVEN (but 2 and 4) states $[6,8,10, \ldots]$, a SuperState $\mathrm{SS}_{2}$ made of the infinite ODD states $[3,5,7, \ldots]$

The matrix $P$ can be partitioned into 6 submatrices, written simply as

$$
P=\left[\begin{array}{ccc}
P_{00} & 0 & 0 \\
P_{10} & P_{11} & P_{12} \\
P_{20} & P_{21} & P_{22}
\end{array}\right]
$$

where $\mathrm{P}_{00}, \mathrm{P}_{11}$ and $\mathrm{P}_{22}$ are square matrices.

Notice that the submatrix $\mathrm{P}_{00}$ is orthogonal: its inverse is its transpose $P_{11}{ }^{-1}=P_{11}{ }^{T}$.

It is important to notice that $\mathrm{P}^{3}$, the $3^{\text {rd }}$ power of the matrix $\mathrm{P}$, is such that the submatrix

$$
P_{00}{ }^{3}=\left[\begin{array}{lll}
1 & 0 & 0 \\
0 & 1 & 0 \\
0 & 0 & 1
\end{array}\right]
$$

is the identity matrix; when the system reaches the set $\{1,2$, 4) (Collatz Cycle) of the states it remains there forever. It follows that $P_{00}{ }^{-1}=P_{00}{ }^{2}=P_{00}{ }^{T}$.

The matrices $\mathrm{P}_{00}, \mathrm{P}_{11}$ and $\mathrm{P}_{22}$ are square matrices, while the others are rectangular; the rectangular submatrices $P_{01}$ and $P_{02}$ in the upper right corner have only 0 entries.

The process is bound to enter the SuperState $\mathrm{SS}_{0}=\{1,2$, 4 ) because the rectangular submatrix $P_{10}$ in the middle left corner has only one 1 entry [the other entries are all 0 ] and the rectangular submatrix $P_{20}$ in lower left corner has only 0 entries. The "periodic process" circulating in the set $\{1,2$, 4 \} is ruled by the submatrix $\mathrm{P}_{00}$.

The graph of the transitions is given in figure 6 .

In the figure 7 we show the flow graph of the 3 SuperStates $\mathbf{S S}_{\mathbf{0}}, \mathbf{S S}_{\mathbf{1}}$ and $\mathbf{S S}_{\mathbf{2}}$ (of the merged process) and the transitions between them; notice that there are three arrows from $\mathbf{S S}_{\mathbf{1}}$, one back to $\mathbf{S S}_{\mathbf{2}}$, one forward to $\mathbf{S S}_{\mathbf{0}}$ and one re-entering into $\mathbf{S S}_{\mathbf{1}}$ (which accounts for the internal 
transitions within $\mathbf{S S}_{\mathbf{1}}$ ).

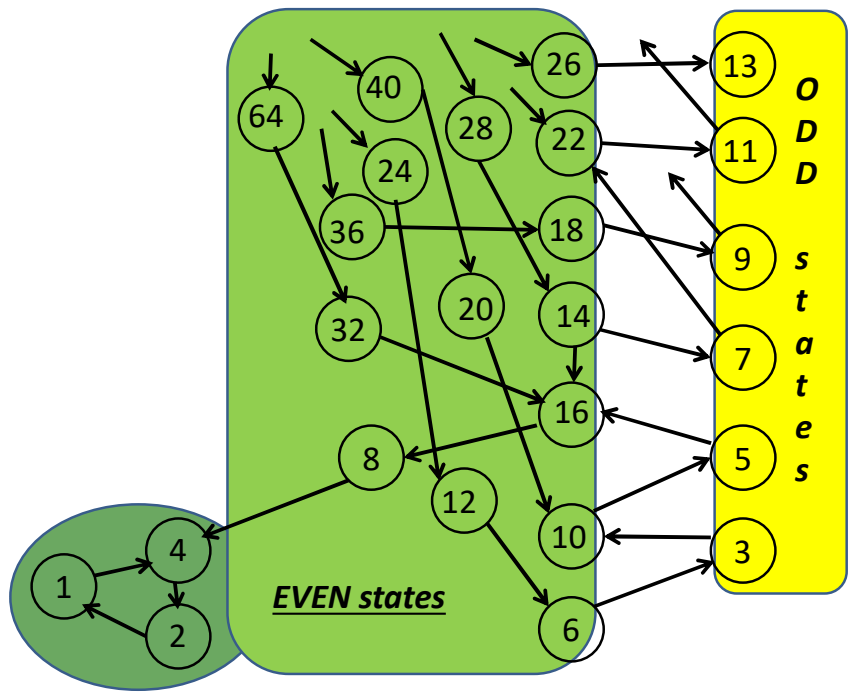

Figure 6. The graph of the transitions within and between the SuperStates $\mathbf{S S}_{\mathbf{0}}, \mathbf{S S}_{\mathbf{1}}$ and $\mathbf{S S}_{\mathbf{2}}$ (only few of the total transitions are shown)

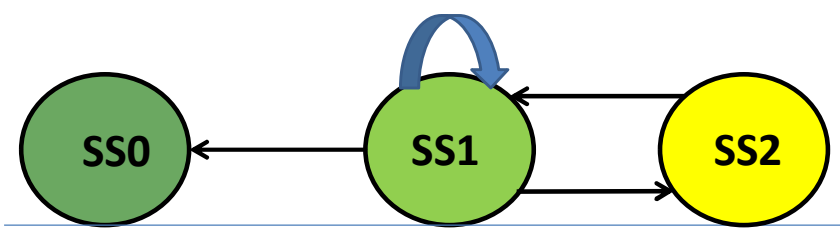

Figure 7. The graph of the transitions (of the merged process) between the SuperStates $\mathbf{S S}_{\mathbf{0}}, \mathbf{S S}_{\mathbf{1}}$ and $\mathbf{S S}_{\mathbf{2}}$

The merged process is ruled by a matrix $\mathrm{P}_{\text {merged }}$ as the following

$$
P_{\text {merged }}=\left[\begin{array}{ccc}
1 & 0 & 0 \\
p_{10} & p_{11} & 0.5 \\
0 & 1 & 0
\end{array}\right]
$$

where the transition probabilities are shown (we shall see later how to find the probabilities $p_{10}$ and $p_{11}$ ).

IF $p_{10}>0$, the matrix $\mathrm{P}_{\text {merged }}$ provides the "steady state probability vector" $\pi=[1,0,0]$ solution of the relationship $\pi=\pi \mathbf{P}_{\text {merged }}$, which states that the process stays forever in the SuperState $\mathbf{S S}_{\mathbf{0}}$ after entering it.

After entering $\mathbf{S S}_{\mathbf{0}}$ the probability of being in the states [making the SuperState $\mathbf{S S}_{\mathbf{0}}$ ] $\{1,2,4\}$ (Collatz cycle) is given by the "steady state probability vector" $\pi^{*}=[1 / 3,1 / 3$, $1 / 3$ ] solution of the relationship $\pi *=\pi * \mathbf{P}_{\mathbf{0 0}}$.

IF $p_{10}>0$, another way of finding the "steady state probability vector" is by using the theory given in the books $[7,8]$ related to Reliability Integral Theory $[R I T]$; one can find two vectors $z \alpha_{1}$ and $z \alpha_{2}$ defined, as follows,

- $z \alpha_{1}$ is the vector of the (steady state) probabilities of entering into into the SuperState $\mathbf{S S}_{\mathbf{0}}$, when there is a transition $\mathbf{S S}_{\mathbf{1}}=\{$ even states $\} \Rightarrow \mathbf{S S}_{\mathbf{0}}=\{1,2,4\}$

- $\mathrm{z \alpha}_{2}$ is the vector of the (steady state) probabilities of entering into the SuperState $\mathbf{S S}_{\mathbf{1}}=\{$ even states $\}$ coming from $\mathbf{S S}_{\mathbf{0}}$.

$\mathrm{z} \alpha_{1}$, in the case of the merged process, is by definition a one-dimensional row vector [1] (which is by the way the $1^{\text {st }}$ entry of the vector $\pi=[1,0,0])$ related to the SuperState $\mathbf{S S}_{\mathbf{0}}$

$\mathrm{zO}_{2}$, in the case of the merged process, is by definition a two-dimensional row vector $[0,0]$ (which is by the way the last two entries of the vector $\pi=[1,0,0])$ related to the SuperState $\mathbf{S S}_{\mathbf{1}}$ and $\mathbf{S S}_{\mathbf{2}}$.

Now we need only to find the value of the probabilities $p_{10}$ and $p_{11}$.

Let's start with $p_{11}$ and look at the matrix (re-arranged from figure 1) in particular to the infinite square submatrix $P_{11}$. Let's assume, for a while, that the dimension are a finite couple $(2 \mathrm{~m}, 2 \mathrm{~m})$; the number of 1 entries in $P_{11}$ are $(\mathrm{m}-1)$; then the ratio, the probability $p_{11}$, is $(\mathrm{m}-1) / 2 \mathrm{~m}=0.5-1 / 2 \mathrm{~m}>$ $0.5-1 /[2(81 \mathrm{~m})]$. The matrix $P_{12}$, for a while, can have the same dimension $(2 \mathrm{~m}, 2 \mathrm{~m})$.

IF the initial condition $\mathrm{y}_{0}$ (any integer positive number) is an odd number, then the $1^{\text {st }}$ iteration provides an even number $3 \mathrm{y}_{0}+1=\mathrm{m}$; we decide to give to the matrices $P_{11}, P_{12}$, $P_{21}, P_{22}$, the dimension $(2 \mathrm{~m}, 2 \mathrm{~m})$; in order to take into account the possibility that, at some iteration $\mathrm{k}$, there could be a transition $y_{k-1} \rightarrow y_{k}$, with $y_{k}>2 m$, we choose for the above 4 matrices a dimension $\mathrm{m}^{\prime}=2\left(3^{4} \mathrm{~m}\right)=2(81 \mathrm{~m})$; finally we set $p_{11}=0.5-1 /[2(81 \mathrm{~m})]$.

It follows that $p_{10}=1 /[2(81 \mathrm{~m})]$.

This argument can be repeated for any finite initial condition $\mathrm{y}_{0}$. We always compute a probability $p_{10}>0$ and we are certain that the "steady state probability vector" $\pi=[1,0,0]$ solution of the relationship $\pi=\pi \mathbf{P}_{\text {merged }}$ exists

The merged process stays forever in the SuperState $\mathbf{S S}_{\mathbf{0}}$ after entering it.

For any $\mathrm{k}$ the iterated value $\mathrm{y}_{\mathrm{k}}$ cannot go to infinity, as proved in [3 by Carletti et al.]

\section{The 3 SuperStates Reliability Model}

Now we consider our Gedanken Experiment [the "ideal machine" which makes transitions between the SuperStates (fig, 7), as quickly as one wants, and finally ends into $\mathrm{SS}_{0}$ the "Collatz Cycle" where it stays forever].

The transitions are ruled by transition rates $\lambda_{1}$ (from $\mathrm{SS}_{2}$ to $\mathrm{SS}_{1}$ ), $\mu$ (from $\mathrm{SS}_{1}$ to $\mathrm{SS}_{2}$ ), $\lambda_{0}$ (from $\mathrm{SS}_{1}$ to $\mathrm{SS}_{0}$ ); the transition rates are not probabilities!

Let's see how our "ideal machine" works: when it is $\mathrm{SS}_{2}$ it goes next into $\mathrm{SS}_{1}$ after a random time ruled by the density transition probability $\lambda_{1} \exp \left(-\lambda_{1} \mathrm{t}\right)$ while when it is $\mathrm{SS}_{1}$ arises a competition between the random time $\mathrm{T}_{0}$, ruled by the density transition probability $\lambda_{0} \exp \left(-\lambda_{0} \mathrm{t}\right)$ [to go to $\mathrm{SS}_{0}$ ] and the random time $\mathrm{T}_{2}$, ruled by the density transition probability $\mu \exp (-\mu \mathrm{t})$ [to go to $\mathrm{SS}_{2}$ ]; if $\mathrm{T}_{0}<\mathrm{T}_{2}$, then the machine goes to $\mathrm{SS}_{0}$ (on the contrary it goes back to $\mathrm{SS}_{2}$ ).

According the Reliability Integral Theory [7, 8] we define the functions $\mathrm{R}_{2}(\mathrm{t})$ and $\mathrm{R}_{1}(\mathrm{t})$ as the probabilities that the machine does not enter $\mathrm{SS}_{0}$ (the Collatz Cycle) at time t, GIVEN that it started in $\mathrm{SS}_{2}$ or $\mathrm{SS}_{1}$, respectively, at time 0 (when the machine begins its operation, that is when we chose $\mathbf{y}_{\mathbf{0}}$ ). 
The two integral equations providing the probabilities $\mathrm{R}_{2}(\mathrm{t})$ and $\mathrm{R}_{1}(\mathrm{t})$ are

$$
\begin{aligned}
& R_{2}(t)=e^{\left(-\lambda_{1} t\right)}+\int_{0}^{t} \lambda_{1} e^{\left(-\lambda_{1} r\right)} R_{1}(t-r) d r \\
& R_{1}(t)=e^{\left[-\left(\lambda_{0}+\mu\right) t\right]}+\int_{0}^{t} \mu e^{\left[-\left(\lambda_{0}+\mu\right) r\right]} R_{2}(t-r) d r
\end{aligned}
$$

The solution $\mathrm{R}_{2}(\mathrm{t})$ of the RIT integral equations $[7,8]$ is

$$
R_{2}(t)=\frac{s_{2}}{s_{2}-s_{1}} \exp \left(-s_{1} t\right)+\frac{s_{1}}{s_{1}-s_{2}} \exp \left(-s_{2} t\right)
$$

where $s_{1}$ and $s_{2}$ are the absolute values of the negative roots of the characteristic equation

$$
s^{2}+s\left(\lambda_{1}+\lambda_{0}+\mu\right)+\lambda_{1} \lambda_{0}=0 .
$$

Since $-\mathrm{s}_{1}$ and $-\mathrm{s}_{2}$ are negative (whichever are the positive parameters $\lambda_{0}, \lambda_{1}$ and $\left.\mu\right) \mathrm{R}_{2}(\mathrm{t}) \rightarrow 0$ as $\mathrm{t} \rightarrow \infty$ : the Collatz Cycle (the superstate $\mathrm{SS}_{0}$ ) is eventually entered as time flows on.

The Mean Time To the SuperState $\mathrm{SS}_{0}$ (from the SuperState $\left.\mathrm{SS}_{2}\right)$ is $\mathrm{MTTSS}_{0}=\left(\lambda_{1}+\lambda_{0}+\mu\right) / \lambda_{1} \lambda_{0}$.

The rates refer to the number of transitions between the superstates, per unit time: it is the speed of our ideal machine. Since the speed is at our will we can fix $\lambda_{1}$ as we want; then $\mathrm{MTTSS}_{0}$ can be as short as we want (provided that the other constraints are respected)!

For example, let's fix $\lambda_{1}=1 / \mathrm{ns}, 1$ transition per nanosecond from $\mathrm{SS}_{2}$ to $\mathrm{SS}_{1}$ which mean a mean time $1 / \lambda_{1}=1 \mathrm{~ns}$.

This consequence of this choice is that the mean time $1 /\left(\lambda_{0}+\mu\right)$ for any transition out of the susperstate $\mathrm{SS}_{1}$ (from $\mathrm{SS}_{1}$ to $\mathrm{SS}_{2}$ and from $\mathrm{SS}_{1}$ to $\mathrm{SS}_{0}$ ) and plus the mean time between transitions within the susperstate $\mathrm{SS}_{1}$ must be $1 \mathrm{~ns}$.

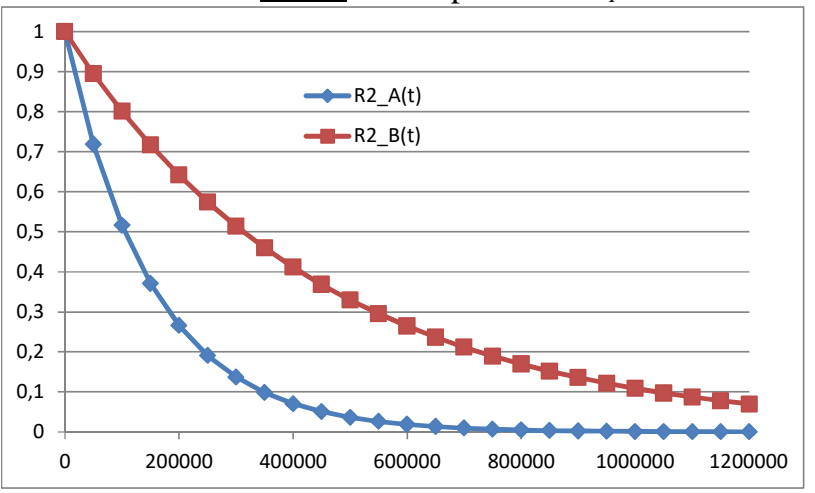

Figure 8. The "Reliabilities" associated to the SuperState $\mathbf{S S}_{\mathbf{2}}$, for two initial values $\mathrm{y}_{0}$ and $\mathrm{y}_{0}{ }_{0}\left(\mathrm{y}_{0}<\mathrm{y}_{0}{ }_{0}\right)$

To be consistent with the matrix $\mathrm{P}_{\text {merged }}$ the "steady state" transition probability $p_{12}=\mu /\left(\lambda_{0}+\mu\right)=0.5$ from $\mathrm{SS}_{1}$ to $\mathrm{SS}_{2}$.

The transition rate $\lambda_{0}$ is related to the transition probability $\mathrm{p}_{10}$ of the $\mathrm{P}_{\text {merged }}$ matrix: $p_{10}=\lambda_{0} /\left(\lambda_{0}+\mu\right)$.

The ratio $p_{10} / p_{12}=\lambda_{0} / \mu$ provides $p_{10}=\left(\lambda_{0} / \mu\right) 0.5$; if we put $\mu=0.5$ transitions/ns, the machine, in mean, takes 2 ns to go from $\mathrm{SS}_{1}$ to $\mathrm{SS}_{2}$.

We can derive $\lambda_{0}=\mu p_{10} /\left(1-p_{10}\right)$. Previously we set $p_{10}=1 /[2(81 \mathrm{~m})]$, where $\mathrm{m}=3 \mathrm{y}_{0}+1$ with the initial condition $\mathrm{y}_{0}$ (any integer positive odd number).

The figure 8 show two cases.
The Mean Time To $\mathrm{SS}_{0}$ (from $\mathrm{SS}_{2}$ ) are respectively (area under the curves) $151108 \mathrm{~ns}$ (with $\mathrm{y}_{0}$ ) and $451108 \mathrm{~ns}$ (with $\left.\mathrm{y}^{\prime}{ }_{0}\right)$ and $\left(\mathrm{y}_{0}<\mathrm{y}_{0}{ }_{0}\right)$

Having applied to an "ideal machine" (Gedanken Experiment) the SPQR ( Semper Paratus ad Qualitatem et Rationem») Principle and Reliability Integral Theory, the author thinks that his $\underline{\text { new }}$ probabilistic method is able to provide the proof of the Collatz-Syracuse Conjecture [he did already probabilistically in previous papers].

The "ideal machine" can be as fast as one wants, to go from the initial state $\mathrm{y}_{0}$ to the superstate $\mathrm{SS}_{0}$ : it all depends on the transition rate $\lambda_{1}$ and on the mean time $1 / \lambda_{1}$.

All this does not mean that there cannot be at least one number $y_{0}$ that keeps the machine circulating forever between the superstates $S S_{2}$ and $S S_{1}$.

\section{The NON-Probabilistic Model}

It is based on a graph $\mathrm{G}$ comprising two superstates A and B of our system:

- A comprises the states 1, 2, 4

- B comprises all the other states $3,5,6, \ldots \ldots$.

For such a "process" we can draw the diagram (figure 9), where the submatrices are the ones given before [assuming, for the time being, that the back transition $\mathrm{A} \rightarrow \mathrm{B}$ can happen]

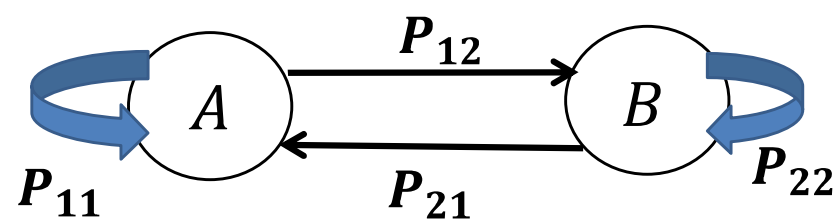

Figure 9. Transition diagram of the graph $\mathrm{G}$

To solve our problem we introduce the concept of generating function.

Let's consider a function $\mathrm{f}(\mathrm{n}), \mathrm{n}=0,1,2, \ldots ., \infty$.

The generating function $F(z)$, written as $G[f(n)]=F(z)$, is the complex function given by

$$
F(z)=\sum_{n=0}^{\infty} f(n) z^{n}
$$

where $\mathrm{z}$ is the complex variable $\mathrm{z}=\mathrm{x}+\mathrm{iy}$. [actually in some documents one finds that $\mathrm{z}^{\mathrm{n}}$ is $\left.\mathrm{z}^{-\mathrm{n}}\right]$. For a finite sequence it is the "characteristic polynomial" of the equation $\mathrm{f}(\mathrm{k})+\mathrm{f}(\mathrm{k}-1)+\ldots .+\mathrm{f}(1)+\mathrm{f}(0)=0$

One important property of $F(z)$ is that $G[f(n-k)]=z^{k} F(z)$.

We can apply the z-transform (the generating function) to the graph of the Siracuse_Collatz sequence (1); we name $\mathrm{n}=0$ the "starting time point" of the sequence which has $\mathrm{y}(0)$, a chosen integer number $\mathrm{y}_{0}$, as the state of the system: let be $\mathrm{y}(0)=\mathrm{y}_{0}$; the initial state is related to the infinite row vector indicated by $\mathrm{u}_{\mathrm{C}}(0)$, of the matrix $\mathrm{P}$ [which, at this time, is not a probability matrix].

Since, according to $(6), \mathrm{u}(\mathrm{k}+1)=\mathrm{u}(\mathrm{k}) \mathrm{P}$, we can find the generating transform

$$
U(z)=z U(z) P
$$


of the sequence $\mathrm{y}_{\mathrm{k}}$ (of the hailstone numbers).

From figure 9 we can draw the flow-graph, with the $\mathrm{z}$-transform, of the process (figure 10)

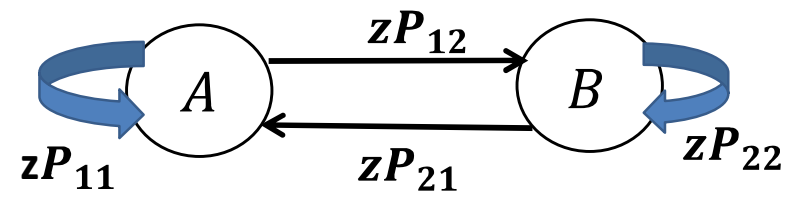

Figure 10. Flow-graph (z transform) of the graph G

Let $\Phi(\mathrm{z})$ the transform of the functional relationship (6).

From (18) we derive

$$
\Phi(z)=U(z)=[I-P z]^{-1}
$$

which solves completely our problem.

Let name the function $\Phi_{\mathrm{i}, \mathrm{j}}(\mathrm{z})$ the transmission from the state $i$ and the state $j$ of our system.

For example, if $\mathrm{i}=5$ and $\mathrm{j}=23$, the transmission $\Phi_{5,23}(\mathrm{z})$ represent transform of the infinite vector (sequence) of values $\left\{\varphi_{5,23}(0), \varphi_{5,23}(1), \ldots, \varphi_{5,23}(\mathrm{n}), \ldots, \varphi_{5,23}(\infty)\right\}$, where we can tell that there can be a value 1 , somewhere, in the sequence; if $\mathrm{i}=5$ and $\mathrm{j}=16$, we know that $\varphi_{5,16}(0)=1 \quad$ [see the matrix P], which means that the states 5 and 16 are connected at step 0 ; are they connected at some other step?

We can partition the matrix $\Phi(\mathrm{z})=\left[\Phi_{\mathrm{i}, \mathrm{j}}(\mathrm{z})\right]$ according the partitioning of the matrix $\mathrm{P}$ (flow graph of figure 10), getting

$$
\Phi(\mathrm{z})=\left[\begin{array}{ll}
\Phi_{A, A} & \Phi_{A, B} \\
\Phi_{B, A} & \Phi_{B, B}
\end{array}\right]
$$

where $\Phi_{\mathrm{A}, \mathrm{A}}(\mathrm{z})$ is the transmission between the various states in the set $A, \Phi_{A, B}(z)$ is the transmission from the various states of the set $A$ to the set $B, \Phi_{B, A}(z)$ is the transmission from the various states of the set $B$ to the set $A$ and $\Phi_{B, B}(z)$ is the transmission between the various states in the set $\mathrm{B}$.

Let now consider the transmissions $\Phi_{\mathrm{i}, \mathrm{j}}(\mathrm{z})$ with $\mathrm{i} \in \mathrm{B}$ and $\mathrm{j} \in \mathrm{B}$; they collectively provide all the transitions of the Collatz process before entering the Collatz cycle $A=[1,2,4]$ where it stays forever ("periodic process").

We consider the following flow-graph (figure 11, where the upper oval is RED and the down oval is GREEN)

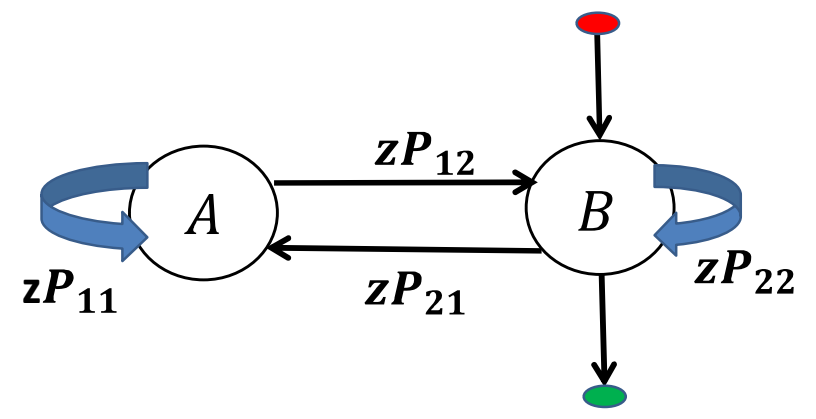

Figure 11. The flow-graph when we consider the transmissions from the read state to the green state, both in the same set B

With the theory we have

$$
\Phi_{\text {red,green }}(\mathrm{z})=z P_{21}\left[I-z P_{11}\right]^{-1} z P_{12}
$$

Looking at the matrix $\mathrm{P}$ and its partition, it is clear that

$$
\Phi_{\text {red,green }}(\mathrm{z})=[0]
$$

which means that only one of the subsequent transmissions $\varphi_{\text {red,green }}(n)$ for $n=0$ to $n \rightarrow \infty$ can be 1 , while all the others are 0

$$
\begin{gathered}
\left\{\varphi_{\text {red,green }}(0), \varphi_{\text {red,green }}(1), \ldots, \varphi_{\text {red,green }}(\mathrm{n}), \ldots, \varphi_{\text {red,green }}(\infty)\right\}= \\
\{0,0, \ldots, 0, \ldots, 0\}
\end{gathered}
$$

unless, at step 0 , the states red and green are chosen to satisfy

$$
y_{1}[\text { green }] \quad\left\{\begin{array}{cccc}
=\frac{1}{2} y_{0} I F & y_{0}[\boldsymbol{r e d}] \text { is } E V E N \\
=3 y_{0}+1 & I F & y_{0}[\boldsymbol{r e d}] \text { is } O D D
\end{array}\right\}
$$

It follows that the process [Collatz] is such that it cannot circulate within the set B forever.

Therefore it enters, at some step, the Collatz cycle $\mathrm{A}=[1$, 2, 4] ("periodic process"), where it stays forever.

This last statement is a non-probabilistic proof: the Collatz cycle $A=[1,2,4]$ is bound to be entered, whichever is the initial integer number $\mathrm{y}_{0}$.

The probabilistic proofs of the other sections state that the entering of the Collatz cycle $A=[1,2,4]$ has probability 1.

\section{Conclusion}

Having applied the SPQR («Semper Paratus ad Qualitatem et Rationem») Principle, the author thinks that his methods either probabilistic or non_probabilistic are able to show the proof of the Collatz-Syracuse Conjecture.

The last method, based on the flow-graph theory, provided us the means to solve completely the problem.

\section{REFERENCES}

[1] http://maddmaths.simai.eu/category/rubriche/fake_papers/

[2] Paul S. Bruckman A proof of the Collatz conjecture, International Journal of Mathematical Education in Science and Technology 39 (2008), no. 3, 403-407

[3] T. Carletti, D. Fanelli, 2016, Quantifying the degree of average contraction of Collatz orbits. arXiv:1612.07820.v1

[4] J.C. Lagarias, "The Ultimate Challenge: the $3 x+1$ problem". American Mathematical Society. (2010).

[5] J.C. Lagarias, The $3 x+1$ problem annotated bibliography, 1995

[6] Galetto, F., 2019, Proof of the Syracuse_Collatz Conjecture. 2019. <hal-02048821> and Academia.Edu

[7] Galetto, F., 2016, Reliability and Maintenance, Scientific Methods, Practical Approach, Vol-1, www.morebooks.de.

[8] Galetto, F., 2016, Reliability and Maintenance, Scientific Methods, Practical Approach, Vol-2, www.morebooks.de. 
[9] Galetto, F., 2017, The SPQR ( $\ll$ Semper Paratus ad Qualitatem et Rationem») Principle in Action. Engineering and Applied Sciences.

[10] Galetto, F., 2019, Syracuse_Collatz Conjecture: Comparison of two Markov approaches towards the proof, 2019. <hal-02274552> and Academia.Edu

[11] Galetto, F., 2019, Proof of Syracuse_Collatz Conjecture by Flow Graphs, <hal-02288698> and Academia.Edu

[12] Galetto, F., 2019, Collatz Conjecture, new proof by merging into three SuperStates <hal-02316263> and Academia.Edu
[13] Galetto, F., 2019, Syracuse_Collatz Conjecture, proof by Reliability Integral Theory <hal- 02316263> and Academia.Edu

[14] Schorer, P., The Remarkably Simple Structure of the $3 x+1$ Function

[15] Schorer, P., A Solution to the $3 x+1$ Problem, occampress.com

[16] Schorer, P., A Possible Reason Why Mathematicians Are Unwilling to Read My Paper, "A Solution to the $3 x+1$ Problem", on occampress.com 